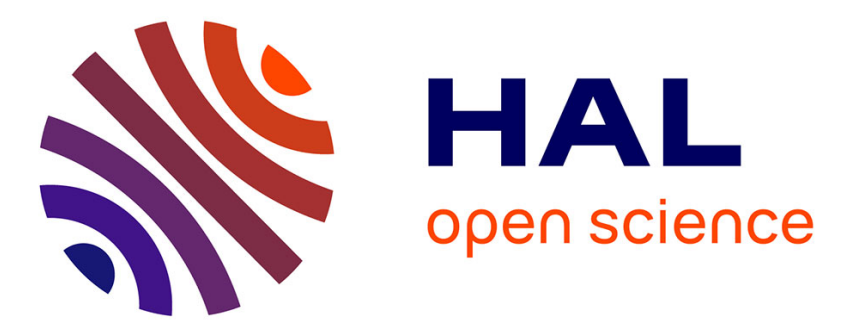

\title{
Determining the exit time distribution for a closed cyclic network
}

\author{
François Baccelli, William A. Massey, Paul E. Wright
}

\section{To cite this version:}

François Baccelli, William A. Massey, Paul E. Wright. Determining the exit time distribution for a closed cyclic network. [Research Report] RR-0832, INRIA. 1988. inria-00076893

\section{HAL Id: inria-00076893 https://hal.inria.fr/inria-00076893}

Submitted on 29 May 2006

HAL is a multi-disciplinary open access archive for the deposit and dissemination of scientific research documents, whether they are published or not. The documents may come from teaching and research institutions in France or abroad, or from public or private research centers.
L'archive ouverte pluridisciplinaire HAL, est destinée au dépôt et à la diffusion de documents scientifiques de niveau recherche, publiés ou non, émanant des établissements d'enseignement et de recherche français ou étrangers, des laboratoires publics ou privés. 


\section{Raponoconerionso}

\section{$\mathbf{N}^{\circ} 832$}

\section{DETERMINING THE EXIT TIME DISTRIBUTION FOR A CLOSED CYCLIC NETWORK}

François BACCELLI William A. MASSEY

Paul. E. WRIGHT 


\title{
DETERMINATION DE LA DISTRIBUTION \\ DU TEMPS DE SORTIE D'UN RESEAU \\ DE FILES D'ATTENTE FERME ET CYCLIQUE
}

\author{
François BACCELLI ${ }^{1}$ \\ William A. MASSEY ${ }^{2}$ \\ Paul WRIGHT ${ }^{3}$ \\ ${ }^{1}$ INRIA Sophia-Antipolis, Valbonne 06565, France \\ ${ }^{2}$ AT\&T BELL LABORATORIES, Murray Hill - NJ 07940, U.S.A. \\ ${ }^{3}$ UNIVERSITY of CALIFORNIA, Dept. of Mathematics, Berkeley - CA 94720, U.S.A.

\begin{abstract}
RESUME
On considère un réseau fermé et cyclique de $N+1$ files d'attente où chaque file possède un serveur unique exponentiel. Utilisant la notion de fonction de Bessel sur un treillis, on détermine de manière explicite les probablités de transition de ce réseau jusqu'à la première date où l'une des files se vide. Le calcul de cette probablité de transition fait appel à un groupe de symétries associé au processus, qui est déterminé comme le produit semi-direct de groupes plus simples. On en déduit une caractérisation explicite du spectre du générateur infinitésimal de ce processus aléatoire. On démontre en particulier que lorsque le nombre de noeuds est supérieur à deux et que le nombre de clients dans le réseau est supérieur au nombre de noeuds, le spectre de ce générateur n'est jamais complètement réel.
\end{abstract}

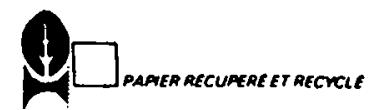




\title{
Determining the Exit Time Distribution for a Closed Cyclic Network
}

Francois Baccelli

INRIA

Domaine de Voluceau Rocquencourt

B. P. 105, 78153 Le Chesnay Cedex, France

William A. Massey

AT\&T Bell Laboratories

Murray Hill, New Jersey 07974

Paul E. Wright

Dept. of Mathematics

University of California

Berkeley, California 94720

\begin{abstract}
Consider a closed, $N+1$-node, cyclic network, where each node bas an independent, exponential single-server. Using lattice-Bessel functions, we can explicitly solve for the transition probabilities of events that occur prior to one of the nodes becoming empty. This calculation entails associating with this absorbed process a symmetry group that is the semi-direct product of simpler groups. As a byproduct, we are able to compute explicitly the entire spectrum for the finite dimensional matrix generator of this process. When the number of nodes exceeds two, and the number of customers in the system is at least one more than the number of nodes, we can show that the total spectrum is never purely real.
\end{abstract}




\section{Determining the Exit Time Distribution for a Closed Cyclic Network}

Francois Baccelli

INRIA

Domaine de Voluceau Rocquencourt

B. P. 105, 78153 Le Chesnay Cedex, France

William A. Massey

AT\&T Bell Laboratories

Murray Hill, New Jersey 07974

Paul E. Wright

Dept. of Mathematics

University of California

Berkeley, California 94720

\section{INTRODUCTION}

Let $N_{\mu}(t)$ be a Poisson process with rate $\mu$. From it we can construct the following multidimensional process on $Z^{N}$, the $N$-dimensional integer lattice. Define $\mathbf{Z}(t)$ to be a randomized random walk on $Z^{N}$, where

$$
\mathbf{Z}(t)=\mathbf{Z}(0)+\sum_{j=0}^{N} N_{\mu j}(t) \mathbf{v}_{j}
$$

The Poisson processes $N_{\mu}(t)$ are independent with possibly distinct rates $\mu_{j}$. If any vector $\mathbf{n}$ in $Z^{N}$ can be written uniquely as $\mathbf{n}=\left(n_{1}, \ldots, n_{N}\right)=\sum_{j=1}^{N} n_{j} \mathbf{e}_{j}$, we define the $\mathbf{v}_{j}$ 's to equal

$$
\mathbf{v}_{j}=\left\{\begin{array}{cl}
\mathbf{e}_{1} & j=0 \\
-\mathbf{e}_{N} & j=N \\
\mathbf{e}_{j+1}-\mathbf{e}_{j} & \text { otherwise }
\end{array}\right.
$$

In Massey [2], it was shown that the transition probabilities for $\mathbf{Z}(t)$ can be written as

$$
P_{\mathrm{w}}(\mathbf{Z}(t)=\mathbf{n})=e^{-(N+1) a t} \boldsymbol{B}^{\mathrm{n}-\mathrm{m}} I(\mathbf{n}-\mathbf{m},(N+1) \gamma t)
$$

where $\alpha$ and $\gamma$ are respectively the arithmetic and geometric means of $\mu_{0}$ thru $\mu_{N}$, $\beta$ equals the following vector

$$
\beta=\left[\frac{\mu_{0}}{\gamma}, \frac{\mu_{0} \mu_{1}}{\gamma^{2}}, \ldots, \frac{\mu_{0} \mu_{1} \cdots \mu_{N-1}}{\gamma^{N}}\right]
$$

$\because: i t^{2} \mathfrak{p}^{\mathrm{n}-\mathrm{m}}=\prod_{j=1}^{N} \beta_{j}^{n_{j}-m_{l}}$, and finally $l(n, \cdot)$ is a lattice Bessel function of rank $N$. These special 
functions were defined by the generating function relation below

$$
\sum_{D \in 2^{N}} x^{D} I(n, y)=\exp \left(\frac{y}{N+1} \sum_{j=0}^{N} x^{v}\right)
$$

where $\mathrm{x}=\left(x_{1}, \ldots, x_{N}\right)$ and the $x_{j}$ 's are non-zero complex numbers.

From this definition, many properties of these lattice-Bessel functions follow, see [2] or [3]. In this paper, we will need only their symmetry properties.

Proposition 1.1. Let $G_{N} \subseteq \operatorname{Aut}\left(Z^{N}\right)$, where

$$
G_{N}=\left\{\pi \mid I(\mathbf{n}, y)=I(\pi(\mathbf{n}), y) \text { for all } \mathbf{n} \in Z^{N}\right\}
$$

We then have

$$
\begin{aligned}
& \text { i. } G_{N} \approx S_{N+1} \text { and is equal to the group of permutations on the set }\left\{v_{0}, \ldots, v_{N}\right\} \\
& \text { ii. } Z_{+}^{N} \text { is a fundamental domain for } G_{N} .
\end{aligned}
$$

In Massey [2], lattice Bessel functions were introduced to solve the exit time problem from the interior of $Z_{+}^{N}$ for the series Jackson network. This was achieved by observing that this problem was equivalent to determining the transition probabilities for the process $Z(t)$ where it is absorbed on the boundary of $Z_{+}^{N}$. The solution is constructed by a symmetry argument, exploiting the fact that $Z_{+}^{N}$ is a fundamental domain for $G_{N}$. From this solution we went on to solve for the joint density of hitting the boundary of $Z_{+}^{N}$ at time $t$, and the state on the boundary that is attained. This was accomplished by using martingale techniques in Baccelli and Massey [1].

In this paper, we determine the analogous transition probabilities for an $N+1$-node, closed cyclic network possessing a fixed number $K$, of customers. This model is equivalent to that of an $N$-node series Jackson network with the added feature of global blocking. New customers are refused entrance into this pipeline system if the current number in there equals $K$. The state space for this process is $\Delta$, where.

$$
\Delta=\left\{\left(n_{i}, \ldots, n_{N}\right) \mid n_{i} \geq 0 . \sum_{j=1}^{N} n_{j} \leq K, \text { and } n_{j} \in Z\right\} .
$$


If we wish to ignore the absorbing states, then our state space can be restricted to $\Delta^{*}$ where

$$
\Delta^{*}=\left\{\left(n_{1}, \ldots, n_{N}\right) \mid n_{j}>0, \sum_{j=1}^{N} n_{j}<K, \text { and } n_{j} \in Z\right\} .
$$

While the state space $\Delta$ is finite compared to $Z_{+}^{N}$, solving for the corresponding absorbing process is more complicated. The notions of group actions, fundamental domains, and semi-direct products of groups all become essential to the exact solution of this problem through the use of lattice-Bessel functions. Moreover, we construct a queueing network model for which the complete spectrum of the generator can be determined. When $N$ exceeds 1 and $K$ exceeds $N+1$, we have examples of transient solutions for Markovian network processes that are not reversible, with spectrum that is not purely real.

\section{SEMI-DIRECT PRODUCT}

Let $G_{N}$ be the Cartesian product of $Z^{N}$ and $G_{N}$ endowed with the following binary operation

$$
\left(\mathrm{m}_{1}, 8_{1}\right) \circ\left(\mathrm{m}_{2}, g_{2}\right)=\left(\mathrm{m}_{1}+g_{1}\left(\mathrm{~m}_{2}\right), 8_{1} 8_{2}\right)
$$

Since $G_{N} \subseteq \operatorname{Aut}\left(Z^{N}\right), G_{N}$ is a group under this operation where $(0, e)$ is the identity, and $(\mathrm{m}, \mathrm{g})^{-1}=\left(-\mathrm{g}^{-1}(\mathrm{~m}), \mathrm{g}^{-1}\right)$. We say that $G_{N}=Z^{N} \times, G_{N}$ is the semi-direct product of $Z^{N}$ and $G_{N}$.

We define $G_{N}^{(0)}$ to be the natural embedding of $G_{N}$ into $G_{N}$ where

$$
G(0)=\left\{\left.(0,8)\right|_{8} \in G_{N}\right\}
$$

For any vector $m$ in $Z^{N}$, we define $G_{N}^{(m)}$ to equal

$$
G_{N}^{(m)}=(\mathbf{m}, e)^{-1} \circ G_{N}^{(0)} \circ(\mathbf{m}, e)
$$

Consider a cyclic closed network with $N+1$ nodes and $K$ customers. We associate with it the following subgroup of $G_{N}$.

$$
G_{K, N}=\left\langle G_{N}^{(0)}, G_{N}^{\left(K v_{0}\right)}\right\rangle
$$


Proposition 2.1. Let $\Lambda$ equal the following sublattice of $Z^{N}$,

$$
\Lambda=\left\{K \cdot \sum_{i=0}^{N} \ell_{i} \mathbf{v}_{i} \mid \ell_{i} \in Z \text { and } \sum_{i=0}^{N} \ell_{i}=0\right\}
$$

We then have

$$
G_{K, N}=\Lambda \times, G_{N}
$$

Proof: Let $H$ equal $\Lambda \times, G_{N}$. Since elements of $G_{N}$ merely permute the $v_{i}$ 's, we can easily show that $\boldsymbol{H}$ is a group. Direct computation shows that $G_{(\ell)}^{(\ell)}$ and $G_{N}^{\left(X v_{0}\right)}$ are subgroups of $\boldsymbol{H}$, hence $G_{K, N} \subseteq H$.

To show the converse, note that any element of $G_{N}^{\left(K v_{0}\right)}$ is of the form $\left(K\left(g\left(v_{0}\right)-v_{0}\right), g\right)$. One special case of the group operation is

$$
\left(\mathrm{m}_{1}, g_{1}\right) \circ\left(0, g_{2}\right)=\left(\mathrm{m}_{2}, g_{1} g_{2}\right)
$$

So applying $\left(0, g^{-1}\right)$ to the right of $\left(K\left(g\left(v_{0}\right)-v_{0}\right), g\right)$ shows that $\left(K\left(v_{t}-v_{0}\right), e\right)$ belongs to $G_{X, N}$ for all $i$. Applying a different $(0,8)$ to the right shows that $\left(K\left(v_{i}-v_{0}\right), g\right)$ belongs to $G_{X, N}$ for all $g$ in $G_{N}$ and all $i$. Letting $g$ equal $e$, and making liberal use of the formula below,

$$
\left(e, m_{1}\right) \circ\left(g_{2}, m_{2}\right)=\left(g_{2}, m_{1}+m_{2}\right)
$$

we see that any element of the form $\left(K \cdot \sum_{i=1}^{N} \ell_{i}\left(v_{t}-v_{0}\right), 8\right)$ belongs to $G_{K, N}$. Defining $\ell_{0}=-\sum_{i=1}^{N} \ell_{i}$, we see that $H \subseteq G_{K, N}$ and so $H=G_{K, N}$.

Let each element of $G_{N}$ act on $Z^{N}$ by defining

$$
(\mathbf{m}, \boldsymbol{g})(\mathbf{n})=\boldsymbol{g}(\mathbf{n})+\mathbf{m} .
$$

The composition law for these mappings is exactly the group operation (2.1) defined for the semidirect product.

Let $\left\{w_{0}, \ldots, w_{N}\right\}$ be a subset of $Z^{N}$ where their integer span, $\left\langle w_{0}, \ldots, w_{N}\right\rangle$ equals $Z^{N}$ and $\sum_{i=0} \mathbf{w}_{j}=0$. We will call such a set a projective basis of $Z^{N}$ and associate with it the following set 
of objects:

$$
\begin{aligned}
\text { i. } & G(w)=\left\{\text { automorphisms on } Z^{N} \text { that permute the } w_{j} \text { 's }\right\} \\
\text { ii. } & \quad \Lambda(w)=\left\{K \sum_{j=0}^{N} \ell_{j} w_{j} \mid \sum_{j=0}^{N} \ell_{j}=0, \text { with } \ell_{j} \in Z\right\} \\
\text { iii. } & \Delta(w)=\left\{\sum_{j=0}^{N} \ell_{j} w_{j} \mid \ell_{0} \geq \cdots \geq \ell_{N}, \ell_{0}-\ell_{N} \leq K, \text { with } \ell_{j} \in Z\right\} \\
\text { iv. } \quad & \Delta *(w)=\left\{\sum_{j=0}^{N} \ell_{j} w_{j} \mid \ell_{0}>\cdots>\ell_{N}, \ell_{0}-\ell_{n}<K, \text { with } \ell_{j} \in Z\right\} .
\end{aligned}
$$

These quantities are related by the following theorem.

Theorem 2.2. Let $\left\{w_{0}, \ldots, w_{N}\right\}$ be a projective basis of $Z^{N}$. We can induce a $G(w)$-action on $Z^{N} / \Lambda(w)$, such that

$$
\Delta(w) \cong\left(Z^{N} / \Lambda(w)\right) / G(w)
$$

Moreover, if $\left(Z^{N} / \Lambda(w)\right)^{*}$ denotes the set of elements in $Z^{N} / \Lambda(w)$ that have full orbits with respect to the $G(w)$-action, then

$$
\Delta *(w) \equiv\left(Z^{N} / \Lambda(w)\right) * / G(w)
$$

Proof: If we set $w_{j}=v_{j}$ for $j=0, \cdots, N$, then $G(w)=G_{N}, \Lambda(w)=\Lambda, \Delta(w)=\Delta$, and $\Delta^{*}(w)=\Delta^{*}$. We will prove the theorem only for this specific case and note that we only use the fact that $\left\{\mathbf{v}_{0}, \cdots, \mathbf{v}_{N}\right\}$ is a projective basis.

To prove these results, it is sufficient to verify the following:

i. For every $n \in Z^{N}$, there exists a $\sigma \in G_{X, N}$ such that $\sigma(n) \in \Delta$.

ii. If $m, n \in \Delta$, then $\sigma(m)=n$ for some $\sigma \in G_{K, N}$ if and only if $m=n$. Moreover, if either $\mathbf{m}$ or $\mathbf{n}$ belongs to $\Delta^{*}$, then $\sigma(m)=\mathbf{n}$ is equivalent to $\sigma=(0, e)$.

If $\theta$ maps each element in $Z^{N}$ to its equivalence class in $\left(Z^{N} / \Lambda\right) / G_{N}$, then (i) and (ii) show that it induces an isomorphism between $\Delta$ and $\left(Z^{N} / \Lambda\right) / G_{N}$. For any $m \in Z^{N}$, by (i) there exists a $\sigma \in G_{\pi^{\prime} N}$ such tha: $\sigma(m) \leqslant \Delta$. Since $\sigma(m)=g(m)+\lambda$ for some $g \in G_{N}$ and $\lambda \in \Lambda$, this means that $\theta(\mathbf{m})=\theta(\mathbf{n})$ for some $\mathbf{n} \in \Delta$. Consequently, $\theta(\Delta)=\left(Z^{N} / \Lambda\right) / G_{N}$ is verified. Moreover, this 
mapping is one-one. Otherwise, there exists a $g \in G_{N}$ and $\lambda \in \Lambda$ such that $m=g(\mathbf{n})+\lambda$ for some $\mathrm{m}, \mathrm{n} \in \Delta$. This gives us $\mathrm{m}=\sigma(\mathrm{n})$ where $\sigma=(\lambda, g) \in G_{X, N}$, which contradicts (ii). Moreover, if $\mathrm{n} \in \Delta$ but $\mathbf{n} \notin \Delta^{*}$, then there exists a non-trival $\sigma \in G_{K_{1} N}$ such that $\sigma(\mathbf{n})=\mathbf{n}$. Either $n_{j}=0$ for some $j=1, \ldots, N$ and $\sigma=(0,8)$ where $g$ permutes only $v_{j-1}$ and $v_{j}$, or $\sum_{j=1}^{N} n_{j}=K$ and $\sigma=\left(K\left(v_{0}-v_{N}\right), g\right)$ where $g$ permutes only $v_{0}$ and $v_{N}$. This cannot hold for $n \in \Delta^{*}$ by (ii), so $\theta$ induces an isomorphism between $\Delta^{*}$ and $\left(Z^{N} / \Lambda\right)^{*} / G_{N}$.

First, we will prove (i). For all $n \in Z^{N}$ we can write it as $\sum_{j=0}^{N} n_{j} v_{j}$. The $n_{j}^{*}$ 's are unique up to adding on a constant to each term. Notice that $n_{0}^{*}-n_{N}^{*}=\sum_{j=1}^{N} n_{j}$ where $n=\sum_{j=1}^{N} n_{j} \mathrm{e}_{j}$. We will say that $n_{0}^{*}-n_{N}^{*}$ equals the length of $n$. For any $n \in Z^{N}$, let $n^{+}=g(n)$ where $g$ is the element of $G_{N}$ that gives a unique $g(n) \in Z_{+}^{N}$. Now we can define a sequence $\left\{m(\ell) \mid \ell \in Z_{+}\right\}$as follows:

1. $\mathbf{m}(0)=\mathbf{n}^{+}$

2. $\mathbf{m}(\ell+1)=\left(\mathbf{m}(\ell)+K\left(\mathbf{v}_{N}-v_{0}\right)\right)^{+}$.

By (2.2), we see that each successive $m(\ell)$ is constructed by applying an element from $\boldsymbol{G}_{K, N}$ to the previous vector. So if $m(\ell) \in \Delta$ bolds for some finite $\ell$, we have mapped an arbitrary element $\mathbf{n}$ of $Z^{N}$ into $\Delta$ using $G_{K, N}$.

Let $a$ be the maximal integer such that $m_{0}^{*}(0)=m_{a}^{*}(0)$ and $m_{N-a}^{*}(0)=m_{N}^{*}(0)$. If the length of $\mathbf{m}(0)$ exceeds $K$, then

$$
\begin{aligned}
& m_{0}^{\dot{1}}(0)=m_{0}^{*}(1)=\ldots=m_{0}^{\dot{*}}(a-1) \\
& m_{N}^{\dot{*}}(0)=m_{N}^{\dot{1}}(1)=\ldots=m_{N}^{*}(a-1)
\end{aligned}
$$

Hence the length of $m(\ell)$ is constant for $\ell=0, \ldots, a-1$. After that, we have

$$
m_{0}^{*}(0)-m_{\lambda}^{*}(0)>m_{i}^{*}(a)-m_{N^{*}}^{*}(a)
$$

Now $a \leq N+1$, sq by induction on the length of a positive vector, there exists some finite $\ell$ such that the length of $m(\ell)$ is less than or equal to $K$. From this we have $m(\ell) \in \Delta$, which finishes the proof of (i). 
For (ii), there exists some $8 \in G_{N}$ by Proposition 2.1, and integers $\ell_{i}$ with $\sum_{i=0}^{N} \ell_{i}=0$ such that

$$
g(\mathbf{m})+K \cdot \sum_{i=0}^{N} \ell_{i} v_{i}=n
$$

We now set $\mathrm{n}=\sum_{i=0}^{N} n_{i}^{*} v_{i}$ with $n_{i}^{*}=\sum_{j=i+1}^{N} n_{j}$ and $g(m)=\sum_{i=0}^{N} g(m)_{i}^{*} v_{i}$ with $g(m)_{i}^{*}=\sum_{j=g^{-1}(i)+1}^{N} m_{j}$.

For some fixed integer $\lambda$, we have the following relations

$$
n_{i}-g(m) i+\lambda=K \ell_{i} \text {. }
$$

Now choose some arbitrary index and call it $i(0)$. We then have for all $i$

$$
n_{i(0)}^{*}-n_{i}^{*}-\left(g(m)_{i(0)}^{*}-g(m)_{i}^{*}\right)=K\left(\ell_{i(0)}-\ell_{i}\right)
$$

First, assume $m$ has length strictly less than $K$, so $0 \leq g(m) i<K$ for all $i$, whereas for the $n_{i}^{*}$ 's we have $0 \leq n_{i} \leq K$. This gives us

$$
\left|\ell_{i(0)}-\ell_{i}\right| \leq \frac{1}{K}\left[\left|n_{i(0)}^{*}-n_{i}^{*}\right|+\left|g(m)_{i(0)}^{*}-g(m)_{i}^{*}\right|\right]<\frac{1}{K}[K+K] \leq 2
$$

Since the $\ell_{i}$ 's are integers, we have $\ell_{i(0)}-\ell_{i}$ equal to $-1,0$, or 1 for all $i$. However,

$$
\ell_{i(0)}=\frac{1}{N+1} \sum_{i=0}^{N} \ell_{i(0)}=\frac{1}{N+1} \sum_{i=0}^{N} \ell_{i(0)}-\ell_{i}
$$

but $\ell_{i(0)}-\ell_{i}=0$ for $i=i(0)$, and $\left|\ell_{i(0)}-\ell_{i}\right| \leq 1$ in general, so we get $\left|\ell_{i(0)}\right| \leq \frac{N}{N+1}$. Thus $\ell_{i(0)}=0$ since $\ell_{i(0)}$ must be an integer. Recall that $i(0)$ was selected arbitrarily, so this proves that $\ell_{i}=0$ for all $i$. Therefore, $g(m)=n$, and the rest follows from the properties of $G_{N}$.

Now suppose that $m, n \in \Delta$ and they both have lengths equal to $K$. Let $b$ be the maximal integer such that

$$
m_{0}^{*}=n_{0}^{*}=\cdots=m_{b}^{*}=n_{b}^{*}=K \text {. }
$$

Given $n=g(m)+\lambda$, we can transform this equation into $\dot{\mathbf{n}}=\dot{g}(\dot{\mathbf{m}})+\dot{\lambda}$, where 


$$
\begin{aligned}
& \dot{\mathbf{n}}=h\left(\mathbf{n}-K \cdot \sum_{j=0}^{b} \mathbf{v}_{j}\right) \\
& \dot{\mathbf{m}}=h\left(\mathrm{~m}-K \sum_{j=0}^{b} \mathbf{v}_{j}\right) \\
& \dot{\bar{g}}=h g h^{-1} \\
& \dot{\lambda}=\lambda+K \cdot \sum_{j=0}^{b} h\left(\mathbf{v}_{j}\right)-v_{j}
\end{aligned}
$$

and finally $h$ is the automorphism from $G_{N}$ that makes $\dot{\mathbf{n}}=\left(\mathbf{n}-\boldsymbol{K} \cdot \sum_{j=0}^{b} \nabla_{j}\right)^{+}$and $\overline{\mathbf{m}}=\left(\mathrm{m}-K \cdot \sum_{j=0}^{b} \mathbf{v}_{j}\right)^{+}$. Both $\dot{\mathbf{m}}$ and $\dot{\mathrm{n}}$ belong to $\Delta$, but by the definition of $b$, one of them does not have a length equal to $K$. Using our previous argument, we get $\dot{\mathbf{m}}=\dot{\mathbf{n}}$ and so $\mathbf{m}=\mathbf{n}$.

A consequence of this theorem is that just as $Z_{+}^{N}$ is a fundamental domain for $G_{N}$, we have now shown that $\Delta$ is a fundamental domain for $G_{X, N}$. This observation yields the following theorem.

Theorem 2.3. Let $T=\inf \{t \mid Z(t) \in \partial \Delta\}$, where $\partial \Delta=\Delta \Delta^{*}$. We then have

$$
P_{\mathrm{m}}(\mathrm{Z}(t)=\mathrm{n}, T>t)=\sum_{\sigma \in G_{K, N}}(-1)^{\sigma} \beta^{\mathrm{n}-\sigma(\mathrm{n})} P_{\mathrm{m}}(\mathrm{Z}(t)=\sigma(\mathrm{n}))
$$

Proof: Let $r_{1}(m, n)$ equal the right hand side of (2.4). If $p_{r}(m, n)=P_{m}(Z(t)=n)$, then let $s_{l}(m, n)$ be defined as follows:

$$
s_{t}(\mathbf{m}, \mathbf{n})=\frac{p_{t}(\mathbf{m}, \mathbf{n})}{\beta^{\mathbf{n}-\mathbf{m}}}=e^{-(N+1) \alpha t} I(\mathbf{n}-\mathbf{m},(N+1) \gamma t)
$$

Given the symmetry of the lattice-Bessel functions, we have for all $8 \in G_{N}$

$$
s_{t}(g(\mathbf{m}), g(\mathbf{n}))=s_{\ell}(\mathbf{m}, \mathbf{n})
$$

and for all $k \in Z^{N}$

$$
s_{t}(\mathbf{m}+\mathbf{k}, \mathbf{n}+\mathbf{k})=s_{t}(\mathbf{m}, \mathbf{n}) .
$$

By these two identities, we have for all $\sigma$ in $G_{K, N}$ 


$$
s_{\imath}(\sigma(\mathbf{m}), \sigma(\mathbf{n}))=s_{1}(\mathbf{m}, \mathbf{n})
$$

Since we are given

$$
r_{r}(\mathbf{m}, \mathbf{n})=\sum_{\sigma \in G_{K, N}}(-1)^{\sigma} \beta^{n-\sigma(n)} p_{t}(\mathbf{m}, \sigma(\mathbf{n}))
$$

the symmetry of the $s_{t}(m, n)$ also gives us

$$
r_{t}(\mathbf{m}, \mathbf{n})=\sum_{\sigma \in G_{K, N}}(-1)^{\sigma} \beta^{\sigma(m)-m} p_{t}(\sigma(\mathbf{m}), \mathbf{n})
$$

By (2.6), $r_{r}(m, n)$ satisfies the same forward equations as the $p_{t}(m, n)$ for the process $Z(t)$. For $n \in \partial \Delta$, there exists an inversion $\sigma \in G_{X, N}\left(\sigma^{2}=e\right)$ such that $\sigma(n)=n$. Using (2.5), it follows that $r_{t}(m, n)=0$ for all $n \in \partial \Delta$. Finally if $t=0$, then $p_{0}(m, n)=\delta_{m, d}$. By Proposition 2.2, we know that $\sigma(m)=n$ if and only if $m=n$ and $\sigma=e$ when $m \in \Delta^{*}$. Therefore $r_{0}(m, n)=\delta_{m, n}$ and by uniqueness, $r_{\mathrm{f}}(\mathrm{m}, \mathrm{n})$ must equal $P_{\mathrm{m}}(\mathrm{Z}(t)=\mathrm{n}, T>t)$.

\section{SPECTRAL DECOMPOSITION}

We will now use this solution to render a more tractable representation. First, we choose the following basis for $Z^{N}$ :

$$
\mathbf{f}_{j}=\left\{\begin{array}{cc}
\mathbf{e}_{j}+j \mathbf{e}_{N} . & j<N \\
\mathbf{e}_{N} . & j=N
\end{array}\right.
$$

For any $m$ in $Z^{N}$, let $m_{N}(f)=m_{N}-\sum_{j=1}^{N-1} j m_{j}$. We then have

$$
\begin{aligned}
\mathrm{m} & =\sum_{j=1}^{N} m_{j} \mathbf{e}_{j}=m_{N}(f) \mathbf{f}_{N}+\sum_{j=1}^{N-1} m_{j} \mathbf{f}_{j} . \\
Z^{N} & =\bigoplus_{j=1}^{N}\left\langle\mathbf{f}_{j}\right\rangle
\end{aligned}
$$

where $\left\langle f_{j}\right\rangle$ is the integer span of $f_{j}$. Since $(N+1) K f_{N}=\sum_{j=0}^{N-1} K\left(v_{j}-v_{N}\right)$ and $K f_{j}=\sum_{k=0}^{j-1} K\left(v_{k}-v_{N}\right)$ for $1 \leq j \leq N-1$, we have 


$$
\dot{\Lambda}=\left(\bigoplus_{j=1}^{N-1}\left\langle K f_{j}\right\rangle\right) \oplus\left((N+1) K I_{N}\right\rangle
$$

From this, it follows that

$$
Z^{N} / \Lambda \cong Z_{X}^{N-1} \oplus Z_{(N+1) X}
$$

Let $T=\left\{e^{1 \theta} \mid 0 \leq \theta<2 \pi\right\}$, which is a multiplicative group, and let $T^{N}$ be its $N$-fold direct sum. Its group operation will be componentwise multiplication. Now define a group homomorphism $\phi$ from $Z^{N}$ to $T^{N}$ where

$$
\phi(m)=\left(\omega_{K}^{m}, \ldots, \omega_{K}^{m_{N}-1}, \omega_{(N+1) K}^{m_{N}(Y)}\right)
$$

with $\omega_{(N+1) K}=\exp \left(\frac{2 \pi i}{(N+1) K}\right)$ and $\omega_{K}=\exp \left(\frac{2 \pi i}{K}\right)$. Identifying a primitive generator of $Z_{(N+1) K}$ with $\omega_{(N+1) X}$, and making a similar association between $Z_{X}$ and $\omega_{X}$, we see that the kernel of the map $\phi$ is precisely $\Lambda$. Thus $\phi$ induces an embedding or monomorphism of $Z^{N} / \Lambda$ into $T^{N}$. Now define $\{m, n\}$ as follows:

$$
\{\mathrm{m}, \mathrm{n}\}=\prod_{j=1}^{N-1} \omega_{X}^{m^{N}} \cdot \omega_{(N+1) K}^{m_{N}(j) m_{N}(j)}
$$

Notice that $\{m, n\}=\left\{m^{\prime}, n^{\prime}\right\}$ when both $m$ and $n$ are congruent to $m$ ' and $n^{\prime}$ respectively, modulo $\Lambda$.

Proposition 3.1. Let $1_{\Lambda}$ be the indicator function for the sublattice $\Lambda$ of $Z^{N}$. We then have

$$
1_{\Lambda}(\mathbf{n})=\frac{1}{\left[Z^{N}: \Lambda\right]} \sum_{\mathbf{m} \in Z^{N / \Lambda}}\{\mathbf{m}, \mathbf{n}\}
$$

Proof: If $n \in \Lambda$, then by (3.2), (3.3), and (3.5), we have $\{m, n\}=1$, and so $1_{\Lambda}(n)=1$. Conversely, if $1_{\Lambda}(n)=1$ then $\left\{m^{\prime}, n\right\}=1$ for all $m^{\prime}$ in $Z^{N}$, since $\left\{m^{\prime}, n\right\}\{m, n\}=\left\{m^{\prime}+m, n\right\}$ and

$$
\left\{m^{\prime}, \mathbf{n}\right\} 1_{\Lambda}(\mathbf{n})=\frac{1}{\left[Z^{N}: \Lambda\right]} \sum_{m \in Z^{N / \Lambda}}\left\{m+m^{\prime}, n\right\}=\frac{1}{\left[Z^{N}: \Lambda\right]} \sum_{m \in Z^{N / \Lambda}}\{m, n\}=1_{\Lambda}(\mathbf{n})
$$

Now if $\left\{m^{\prime}, n\right\}=1$ for all $m^{\prime}$ in $Z^{N}$, let $m^{\prime}=f_{j}$. By (3.5) we have $\left\{f_{j}, n\right\}=\omega_{k}^{n}=1$ for 
$j \leq N-1$, otherwise $\left\{f_{j}, \mathbf{n}\right\}=\omega_{(N+1) K}^{n(f)}=1$. By $(3.4)$, since $\phi(\mathbf{n})=\left(\left\{\mathfrak{f}_{1}, \mathbf{n}\right\}, \ldots\left\{\left\{\boldsymbol{f}_{N}, \mathbf{n}\right\}\right)\right.$, we have $\phi(n)=(1, \ldots, 1)$, which is the identity element of $T^{N}$, and so $n \in \Lambda$.

Moreover, when $1_{\Lambda}(n) \neq 1$, the above argument tells us that $1_{\Lambda}(n)=0$. Otherwise $\left\{m^{\prime}, n\right\}=1$ for all $m^{\prime}$ and this again would imply $1_{\Lambda}(n)=1$.

Proposition 3.2. If $\mathrm{x}=\left(x_{1}, \ldots, x_{N}\right)$, then

$$
\left.\sum_{\lambda \in \Lambda} x^{\lambda} I(\lambda-m, y)=\frac{x^{m}}{R^{N}(N+1)} \sum_{\ell \in Z^{N} / \Lambda}\{\ell, m\} \exp \left(\frac{y}{N+1} \sum_{j=0}^{N}\{\ell, v\}\right\} x^{\nu}\right) .
$$

Proof: Define an associated group homomorphism $\psi$ from $Z^{N}$ to $T^{N}$ as follows:

$$
\psi(m)=\left(\left\{\mathbf{e}_{1}, \mathbf{n}\right\}, \ldots,\left\{\mathbf{e}_{N}, \mathbf{n}\right\}\right)
$$

This mapping has the following property

$$
\{\mathbf{m}, \mathbf{n}\}=\psi(\mathbf{m})^{\mathbf{n}}
$$

where $\psi(m)^{n}$ is the usual $\prod_{j=1}^{N} \psi(m)_{j}^{n j}$. By Proposition 3.1, we have

$$
\begin{aligned}
\sum_{\lambda \in \Lambda} x^{\lambda} I(\lambda-m, y) & =\sum_{\lambda \in Z^{N}} x^{\lambda} 1_{\Lambda}(\lambda) I(\lambda-m, y) \\
& =\sum_{\lambda \in Z^{N}} x^{\lambda+m} 1_{\Lambda}(\lambda+m) I(\lambda, y) \\
& =\sum_{\lambda \in Z^{N}} \frac{x^{\lambda+m}}{\left[Z^{N}: \Lambda\right]} \sum_{\ell \in Z^{N} / \Lambda}\{\ell, \lambda+m\} I(\lambda, y) \\
& =\frac{x^{m}}{\left[Z^{N}: \Lambda\right]} \sum_{\ell \in Z^{N / \Lambda}}\{\ell, m\} \sum_{\Lambda \in Z^{N}} \Psi(\ell)^{\lambda} x^{\lambda} I(\lambda, y)
\end{aligned}
$$

The rest follows from the definition of $\psi(n)$, the fact that $\left[Z^{N}: \Lambda\right]=K^{N}(N+1)$ by $(3.3)$, and the generating function relation for $I(\lambda, y)$.

To simplify the expression for $P_{m}(Z(t)=n, T>t)$, the following quantities will be useful. 


$$
\begin{aligned}
\delta(\ell, n) & =\sum_{g \in G_{N}}(-1)^{\delta}\{\ell, g(n)\} \\
\epsilon(\ell) & =\frac{1}{N+1} \sum_{j=0}^{N}\left\{\ell, v_{j}\right\}
\end{aligned}
$$

We now define the following bilinear form on $Z^{N}$

$$
\langle\mathbf{m}, \mathbf{n}\rangle=\sum_{j=1}^{N-1} m_{j} n_{j}+\frac{m_{N}(f) n_{N}(f)}{N+1}
$$

Note that $\{m, n\}=\exp \left(\frac{2 \pi i}{K}\langle m, n\rangle\right)$. It is with respect to this bilinear form that we want to define an adjoint matrix. For every $g \in G_{N}$ we define $\hat{g} \in \hat{G}_{N}$ to be the unique integral matrix such that

$$
\langle\hat{g}(\mathbf{m}), \mathbf{n}\rangle=(\mathbf{m}, \mathbf{g}(\mathbf{n})\rangle .
$$

By inspection, $\delta(\ell, n)$ and $\epsilon(\ell)$ bave the following properties:

and

$$
\delta(\ell, g(\mathbf{n}))=\delta(\hat{g}(\ell), \mathbf{n})=(-1)^{8} \delta(\ell, \mathbf{n})
$$

$$
\epsilon(\hat{g}(\ell))=\epsilon(\ell)
$$

for all $g \in G_{N}$.

Proposition 3.3. Let $\left\{\hat{\mathbf{v}}_{0}, \ldots, \hat{\mathbf{v}}_{N}\right\}$ be a subset of $Z^{N}$ such that

$$
\hat{\mathbf{v}}_{j}=\left\{\begin{aligned}
\sum_{k=j+1}^{N} \mathrm{f}_{k}, & 0 \leq j \leq N-1 . \\
-\sum_{k=1}^{N} k \mathrm{f}_{k} . & j=N .
\end{aligned}\right.
$$

We then have $\hat{G}_{N}=G(\hat{\mathbf{v}})$ and $\Lambda=\Lambda(\hat{\mathbf{v}})$.

Proof: By inspection, we see that $\left\{\hat{v}_{0}, \ldots, \hat{\mathbf{v}}_{N}\right\}$ is a projective basis. We can show that $\hat{G}_{N}=G(\hat{\mathbf{v}})$ by proving that $\left\{\hat{v}_{0}, \ldots, \hat{\mathbf{v}}_{N}\right\}$ is "dual" to the projective basis $\left\{\mathbf{v}_{0}, \ldots, \mathbf{v}_{N}\right\}$ in the following sense

$$
\left\langle\hat{\mathbf{v}}_{j}, \mathbf{v}_{k}\right\rangle=\frac{-1}{N+1}+\delta_{j, k}
$$

From this and the fact that $g\left(v_{k}\right)=v_{g(k)}$, we have $\hat{g}\left(\hat{v}_{j}\right)=\hat{v}_{g}-1(j)$, since 


$$
\begin{aligned}
\left\langle\hat{g}\left(\hat{v}_{j}\right), \mathbf{v}_{k}\right\rangle & =\left\langle\hat{v}_{j, g}\left(\mathbf{v}_{k}\right)\right\rangle \\
& =\left\langle\hat{v}_{j}, \mathbf{v}_{g}(k)\right\rangle \\
& =\frac{-1}{N+1}+\delta_{j, g(k)} \\
& =\frac{-1}{N+1}+\delta_{g}-1(n), k \\
& =\left\langle\hat{v}_{g}-1\left(n, v_{k}\right\rangle .\right.
\end{aligned}
$$

Thus $\hat{G}_{N}$ permutes the $\hat{v}_{j}$ 's and so $\hat{G}_{N}=G(\hat{v})$.

To compute the $\left\langle\hat{v}_{j}, v_{k}\right\rangle$ 's, we merely decompose the $\hat{\mathbf{v}}_{j}$ and $\mathbf{v}_{k}$ into their orthogonal components in the f-coordinate system. The $\hat{v}_{j}$ 's were defined as such. The $v_{k}$ 's expressed in the f-coordinate system equal

$$
\mathbf{v}_{k}= \begin{cases}\mathbf{f}_{1}-\mathbf{f}_{N}, & k=0 . \\ \mathbf{f}_{k+1}-f_{k}-\mathbf{f}_{N}, & 1 \leq k \leq N-2 . \\ N f_{N}-f_{N-1}, & k=N-1 . \\ -f_{N}, & k=N .\end{cases}
$$

We will do the case $0 \leq j \leq N-1$ and $1 \leq k \leq N-2$, noting that the other cases can be worked out similarly. For $0 \leq j \leq N-1$ and $1 \leq k \leq N-2$, we have

$$
\begin{aligned}
\left\langle\hat{v}_{j}, \mathbf{v}_{k}\right\rangle & =\left\langle\sum_{\ell=j+1}^{N} f_{\ell}, f_{k+1}-f_{k}-f_{N}\right\rangle \\
& =-\left\langle f_{N}, f_{N}\right\rangle+\sum_{\ell=j+1}^{N-1}\left\langle f_{\ell}, f_{k+1}\right\rangle-\sum_{\ell=j+1}^{N-2}\left\langle f_{\ell,} f_{k}\right\rangle \\
& =\frac{-1}{N+1}+\sum_{\ell=j+1}^{N-1} \delta_{\ell, k+1}-\sum_{\ell=j+1}^{N-2} \delta_{\ell, k} \\
& =\frac{-1}{N+1}+\delta_{j, k} .
\end{aligned}
$$

Finally, we note that $\left\{K f_{1}, \ldots, K f_{N-1}, K(N+1) f_{N}\right\}$ is the module basis for $\Lambda(\hat{v})$, hence $\Lambda(\hat{\mathbf{v}})=\Lambda$. 
Now let $\hat{\Delta}=\Delta(\hat{\mathbf{v}})$, and $\hat{\Delta}^{*}=\Delta^{*}(\hat{\mathbf{v}})$. The above proposition, combined with Theorem 2.2 gives us

$$
\hat{\Delta} \equiv\left(Z^{N} / \Lambda\right) / \hat{G}_{N}
$$

If $\left(Z^{N} / \Lambda\right)^{*}$ equals the coset representatives of $Z^{N} / \Lambda$ with full orbits under the $\hat{G}_{N^{-a c t i o n}}$, we also have

$$
\hat{\Delta}^{*} \equiv\left(Z^{N} / \Lambda\right)^{*} / \hat{G}_{N}
$$

Theorem 3.4. Let $T=\inf \{t \mid Z(t) \in \partial \Delta\}$. We then have

$$
P_{\mathrm{m}}(\mathrm{Z}(t)=\mathrm{n}, T>t)=\frac{\mathrm{\beta}^{\mathrm{n}-\mathrm{m}}}{K^{N}(N+1)} \sum_{\ell \in \Delta^{*}} \delta(\ell, \mathrm{m}) \overline{\delta(\ell, \mathrm{n})} \exp (-(N+1)[\alpha-y \in(\ell)] t)
$$

Proof: Combining Theorem 2.3 with (1.1) and Proposition 3.2, we get

$$
\begin{aligned}
\sum_{\sigma \in G_{K, N}}(-1)^{\sigma} \beta^{n-\sigma(n)} P_{m}(Z(t)=\sigma(n)) & =e^{-(N+1) a t} \beta^{n-m} \sum_{\sigma \in G_{K, N}}(-1)^{\sigma} I(\sigma(n)-m,(N+1) \gamma t) \\
& =e^{-(N+1) a t} \beta^{n-m} \sum_{i \in G_{N}}(-1)^{\ell} \sum_{\lambda \in \Lambda} I(g(n)-m+\lambda,(N+1) \gamma t) \\
& =\frac{e^{-(N+1) \alpha r} \beta^{n-m}}{K^{N}(N+1)} \sum_{8 \in G_{N}}(-1)^{8} \sum_{\ell \in 2^{N} / \Lambda}\{\ell, m-g(n)\} \exp \left(\gamma t \cdot \sum_{j=0}^{N}\left\{\ell, v_{j}\right\}\right.
\end{aligned}
$$

We now simplify the summation term,

$$
\begin{aligned}
& \sum_{\delta \in G_{N}}(-1)^{8} \sum_{\ell \in Z^{N} / A}\{\ell, m-g(n)\} \exp \left(\gamma t \cdot \sum_{j=0}^{N}\left\{\ell, v_{j}\right\}\right)=\sum_{\ell \in Z^{N} / A}\{\ell, m\} \overline{\delta(\ell, n)} \exp (\gamma(N+1) t \cdot \in(\ell)) \\
& =\sum_{g \in G_{N}} \sum_{\ell \in \Delta \cdot}\{\hat{g}(\ell), m\} \overline{\delta(\hat{g}(\ell), n)} \exp (\gamma(N+1) r \cdot \epsilon(\hat{g}(\ell))) \\
& =\sum_{\ell \in \Delta \cdot \Omega^{\prime} \in G_{N}}(-1)^{8}\{\ell, g(\mathrm{~m})\} \overline{\delta(\ell, n)} \exp (\gamma(N+1) t \cdot \epsilon(\ell)) \\
& =\sum_{\ell \in \Delta^{*}} \delta(\ell, m) \overline{\delta(\ell, \mathbf{n})} \exp (\gamma(N+1) t \cdot \epsilon(\ell))
\end{aligned}
$$

and the rest follows.

Corollary 3.5. Let $\mathrm{A}$ be the associated Markov generator for the process that acts like $\mathbf{Z}(t)$ in $\Delta^{*}$ 
until it is absorbed by the boundary $\partial \Delta$. We then have that $\mathbf{A}$ is diagonalizable, and its spectrum equals

$$
-\left[\mu_{0}+\ldots+\mu_{N}-\left(\mu_{0} \cdots \mu_{N}\right)^{\frac{1}{N+1}} \sum_{j=0}^{N}\left\{\ell, v_{j}\right\}\right]
$$

where $\ell$ belongs to $\hat{\Delta}^{*}$. These eigenvalues are all real if and only if $N=1$ or $K=N+1$.

Proof: If $\sum_{j=1}^{N} a_{j} e^{b r r}=0$ for all $t \geq 0$ and the $b_{j}$ 's are distinct, then every $a_{j}$ is equal to zero. From this it follows that Theorem 3.4 must give the unique spectral decomposition for the generator A. The fact that each term is of the form $\sum_{j=0}^{M} a_{j} e^{b j r}$ precludes any nilpotent term associated with the Jordan normal form, so the generator must be similar to a diagonal matrix.

To determine when a spectral element is purely real, we note that this is characterized by whether or not $\epsilon(\ell)$ is purely real. When $N=1$, then $\epsilon(\ell)=\cos \left(\frac{\pi \ell}{K}\right)$, so the total spectrum is real for this case. For $K=N+1, \Delta^{*}$ is a singleton state, namely $\{(1, \ldots, 1)\}$. Since $\hat{\Delta}^{*}$ is isomorphic to $\Delta^{*}$, the corresponding spectral element is then the only entry in the generator, which is $-\sum_{j=0}^{N} \mu_{j}$ and is purely real. It remains to treat the cases where $N \geq 2$ and $K \geq N+2$.

Now consider $\ell=\sum_{j=0}^{N} \ell ; \hat{v}_{j}$, where

$$
\ell_{j}^{*}= \begin{cases}N+1 & j=0 \\ N-j & 1 \leq j \leq N\end{cases}
$$

The $\ell_{j}^{*}$ are strictly decreasing in $j$ and $\ell_{N}^{*}-\ell_{0}^{*}=N+1$, so $\ell$ belongs to $\hat{\Delta}^{*}$ precisely when $K \geq N+2$. We will show that the imaginary part of $\epsilon(\ell)$ is always non-zero when $N \geq 2$ and $K \geq N+2$

First, we use (3.5), (3.10) and (3.11) to get 


$$
\begin{aligned}
& \epsilon(\ell)=\frac{1}{N+1} \sum_{j=0}^{N} \omega_{K} \ell^{j-\frac{1}{N+1}} \sum_{i=0}^{N} \ell_{i}^{*} \\
& =\frac{1}{N+1} \omega_{K}^{-\frac{1}{N+1}\left(\frac{N(N+1)}{2}+1\right)}\left(\sum_{j=0}^{N+1} \omega t-\omega_{K}^{N}\right) \\
& =\frac{1}{N+1} \omega_{K}^{-\left(\frac{N}{2}+\frac{1}{N+1}\right)}\left[\frac{1-\omega_{K}^{N+2}}{1-\omega_{K}}-\omega_{K}^{N}\right] \\
& =\frac{1}{N+1} \omega_{X}-\left(\frac{N}{2}+\frac{1}{N+1}\right)\left[\omega_{X}^{\frac{N+1}{2}} \frac{\sin \left(\frac{\pi}{K}(N+2)\right)}{\sin \left(\frac{\pi}{K}\right)}-\omega_{X}^{N}\right] \\
& =\frac{1}{N+1}\left[\omega_{k}^{\frac{1}{2}-\frac{1}{N+1}} \frac{\sin \left(\frac{\pi}{K}(N+2)\right)}{\sin \left(\frac{\pi}{K}\right)}-\omega_{k}^{\frac{N}{2}}-\frac{1}{N+1}\right] \text {. }
\end{aligned}
$$

Now to show that $\operatorname{Im}(\epsilon(\ell)) \neq 0$, we need only prove that

$$
\sin \left(\frac{\pi}{K}\left(1-\frac{2}{N+1}\right)\right) \sin \left(\frac{\pi}{K}(N+2)\right) \neq \sin \left(\frac{\pi}{K}\left(N-\frac{2}{N+1}\right)\right) \sin \left(\frac{\pi}{K}\right)
$$

Observe that $\left(1-\frac{2}{N+1}\right)(N+2)=N-\frac{2}{N+1}$, and since $N \geq 2$ and $K \geq N+2$ hold, we bave

$$
K \geq N+2>N-\frac{2}{N+1}>1>1-\frac{2}{N+1}>0
$$

We can now prove that (3.12) holds by verifying the following lemma,

Lemma 3.6. Given $0 \leq a<x<b \leq \pi$, then

$$
\sin (x) \sin \left(\frac{a b}{x}\right)+\sin (a) \sin (b)
$$

Proof: Let $f(x)=\sin (x) \sin \left(\frac{a b}{x}\right)$. It is a differentiable function on the open interval $(a, b)$.

Moreover, since $f(a)=f(b)=\sin (a) \sin (b)$, by Rolle's Theorem, we can prove our lemma by showing that $f^{\prime}(x)$ has only one zero in $(a, b)$. 
First, we write $f^{\prime}(x)$ as

$$
f^{\prime}(x)=\cos (x) \sin \left(\frac{a b}{x}\right)-\frac{a b}{x^{2}} \sin (x) \cos \left(\frac{a b}{x}\right) .
$$

If we set $g(x)=\frac{\tan (x)}{x}$, then $f^{\prime}(y)=0$ for some $y$ in $(a, b)$ is equivalent to baving $g(y)=g\left(\frac{a b}{y}\right)$. Now suppose that $y \neq \frac{a b}{y}$. We see that $g(x)$ is positive on $\left(0, \frac{\pi}{2}\right)$ and negative on $\left(\frac{\pi}{2}, \pi\right)$, so $y$ and $\frac{a b}{y}$ must both belong to the first or second interval. By Rolle's Theorem, we have $g^{\prime}(z)=0$ for some $z$ in $(a, b)$ with $z * \frac{\pi}{2}$ where

$$
g^{\prime}(x)=-\frac{\tan x}{x^{2}}+\frac{\sec ^{2} x}{x}
$$

If $g^{\prime}(z)=0$, then

$$
z=\sin (z) \cdot \cos (z)
$$

which is equivalent to $2 z=\sin (2 z)$. The formula can only hold when $z=0$, which contradicts the fact that $a<z<b$. Therefore, we must have $y=\frac{a b}{y}$. From this and $a<y<b$, we deduce that $y=\sqrt{a b}$. Hence $\sqrt{a b}$ is the unique zero for $f^{\prime}(x)$ on $(a, b)$, and we have proved our lemma.

Theorem 3.7. If $\mathrm{n} \in \partial \Delta$ such that $\mathrm{n}-\mathrm{v}_{\mathbf{i}} \in \Delta^{*}$, then

$$
P_{\mathrm{m}}(\mathrm{Z}(T)=\mathrm{n}, T \leq t)=\mu_{i} \int_{0}^{1} P_{\mathrm{m}}\left(\mathrm{Z}(s)=\mathrm{n}-\mathrm{v}_{i}, T>s\right) d s
$$

othenwise $P_{\mathrm{m}}(Z(T)=\mathrm{n}, T \leq t)=0$.

Proof: We use exactly the same argument as that in Baccelli and Massey [1], Theorem 1.

We can now solve the Dirichlet problem associated with the generator $\mathbf{A}$ on the domain $\Delta$.

Corollary 3.8. If $\mathrm{n} \in \partial \Delta$ such that $\mathrm{n}-\mathrm{v}_{\mathbf{i}} \in \Delta^{*}$, then 


$$
P_{\mathrm{m}}(\mathrm{Z}(T)=\mathrm{n})=\frac{\gamma \cdot \beta^{\mathrm{n}-\mathrm{m}}}{K^{N}(N+1)^{2}} \sum_{C \in \Delta^{*}} \frac{\delta(\ell, \mathrm{m}) \overline{\delta\left(\ell, \mathrm{n}-\mathrm{v}_{\ell}\right)}}{\alpha-\gamma \cdot \epsilon(\ell)}
$$

otherwise $P_{m}(Z(T)=n)=0$. 


\section{BIBLIOGRAPHY}

[1] Baccelli, F. and Massey, W. A. Calculating Hitring Times for Series Jackson Nerworks, Second International Workshop on Applied Mathematics and Performance/Reliability Models of Computer/Communication Systems, University of Rome II, May 25-29, 1987.

[2] Massey, W. A. Calculating Exit Times for Series Jackson Networks, (to appear in the Journal of Applied Probability).

[3] Massey, W. A. and Wright, P. E. Lattice Bessel Functions (in preparation).

Imprimé en France par

l'Institut National de Recherche en Informatique et en Automatique 
ISSN ORIO-RSEO 